\title{
Cuba y Yucatán en la mirada de una antropóloga
}

\author{
Andrés Fábregas Puig
}

VICTORIA NOVELO, 2009

\section{Etnografía de una migración}

CIESAS, Conaculta, Instituto de Cultura de Yucatán, México, 241 pp.

$\mathrm{L}$ os antropólogos y antropólogas mexicanos no se han distinguido por realizar estudios y reflexiones en sociedades distintas de la de México. No vamos a discutir ahora esa particular característica del quehacer antropológico en nuestro país, pero vale señalarla porque el libro de Victoria Novelo es producto de un minucioso trabajo de campo en la mayor de las Antillas, Cuba. Novelo ha construido una trayectoria como antropóloga que la sitúa entre lo más destacado - por sus contribuciones- de esa disciplina en nuestro país. Es pionera de los estudios acerca de las artesanías, su trabajo al respecto es ya un clásico. También se cuenta entre los antropólogos que iniciaron el estudio de la clase obrera mexicana, particularmente de sus rasgos culturales y de su vida cotidiana. Ha reflexionado sobre la frontera norte y la vida de la población de origen mexicano en los Estados Unidos, y ahora nos ofrece un texto de excelente factura que rebasa su título, para proporcionarnos una amplia reflexión acerca de los lazos de México con el Caribe.

En la "Introducción", la autora narra cómo surgió y se desarrolló la investigación acerca de los yucatecos

\section{Cuba and Yucatan through a Female Anthropologist's Eyes}

Andrés FÁbregas Puig: Universidad Intercultural del Estado de Chiapas, San Cristóbal de las Casas, Chiapas, México

rectoria@unich.edu.mx

Desacatos, núm. 34, septiembre-diciembre 2010, pp. 189-193

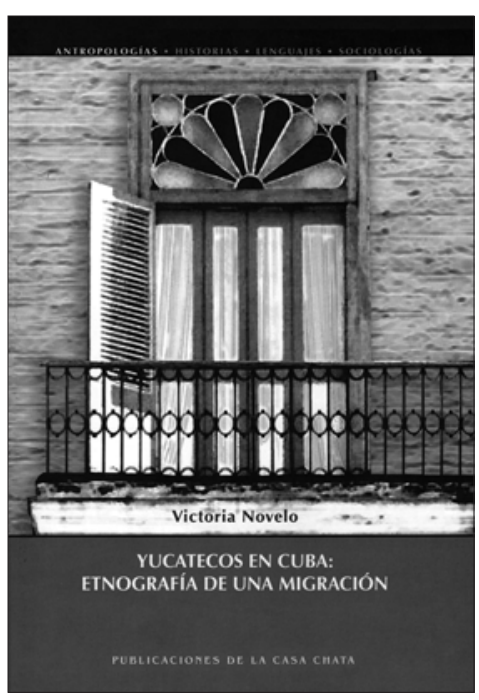

en Cuba, incluyendo la problemática general a la que se enfrentó. En este inicio, un aspecto llamó mi atención: la literatura como fuente para la imaginación antropológica. Como sabemos, Cuba es una tierra de notables escritores, narradores $y$ poetas, la mayoría maestros en el manejo de la palabra. Victoria Novelo abreva en las páginas de Alejo Carpentier, pero también, y aquí se inicia el ejercicio de ligar a Cuba y 


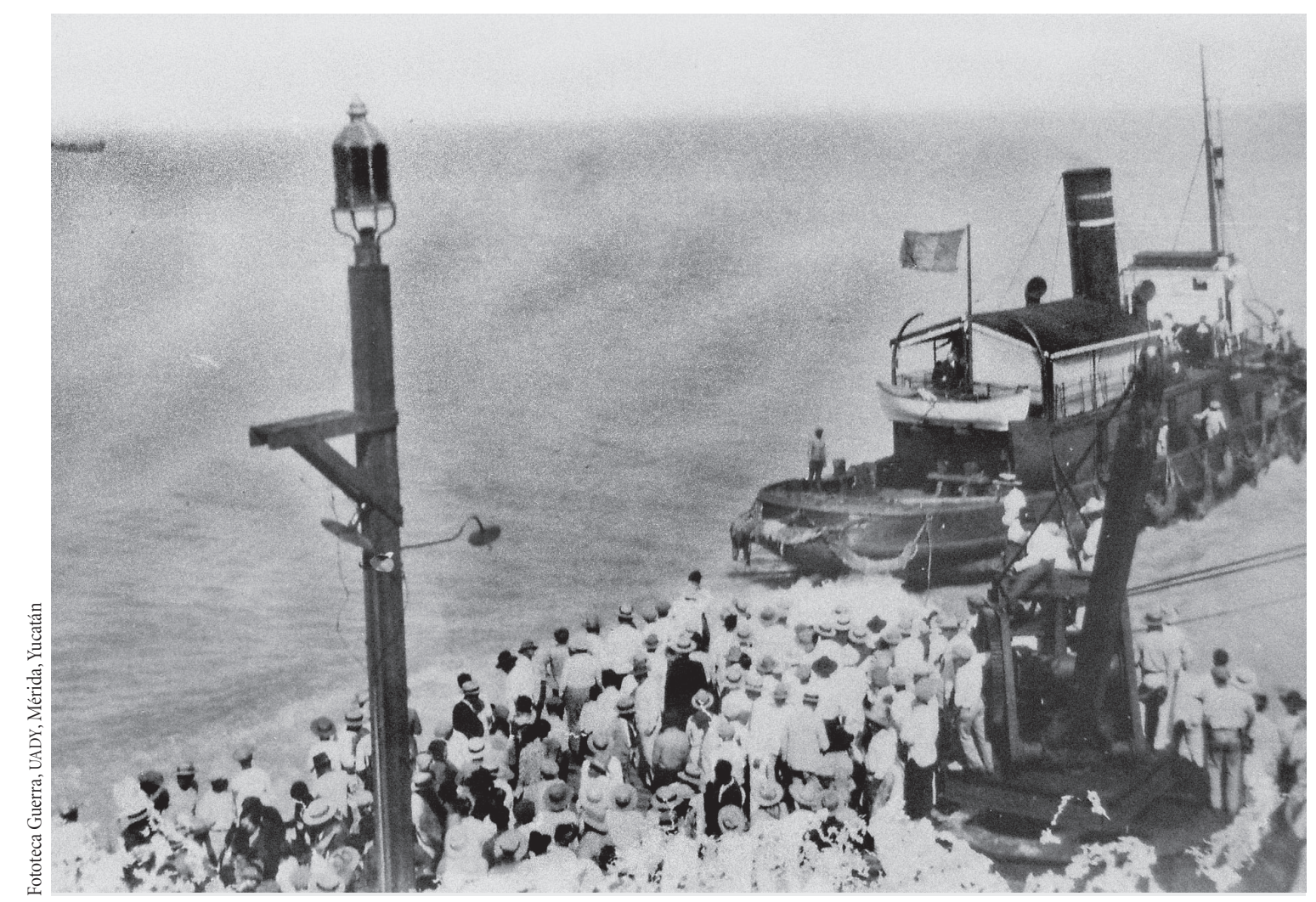

Salidas desde Progreso, ca. 1920.

México, en los textos de la poeta yucateca Rosario Sansores, autora de una columna que se extraña, la cual se publicaba en el periódico Novedades con el título "Rutas de emoción". Es relevante que una antropóloga de la madurez de Victoria Novelo escriba estas experiencias, porque enseñan a las nuevas generaciones la importancia de irse forjando, como en un yunque, en la lectura de la fuente del idioma, que es la literatura, la pasión por la palabra, el vuelco de la imaginación. Novelo asimila la lección de los que miran el mundo desde la ventana de la creación literaria para confabularse con ella y trasladarla a la imaginación antropológica. No es extraño que su texto, como todos sus trabajos, esté bien escrito y transmita un disfrute por el decir bien, con espíritu lúdico a la vez que talante crítico.

La "Introducción" informa de otra influencia no menos importante: la obra de los pioneros de la antropología en México. El asunto es de resaltar en épocas en que la admiración por lo que se escribe en inglés deviene en patología. La a utora se apoya en Manuel Gamio, iniciador de la antropología contemporánea en nuestro país, situando la importancia de su obra sobre los migrantes mexicanos hacia los Estados Unidos. Trabajo pionero, inteligente, el de Manuel Gamio, que debería leerse y discutirse más. Porque allí arranca una tradición de hacer antropología que enfatiza el trabajo de campo, la etnografía, el enfoque regional, la crítica de la situación analizada como método de investigación y la buena escritura como requisito para la presentación de los resultados de investigación y la reflexión. Y aquí también las ligas de México y Cuba se reencuentran en la lectura del pionero cubano, Fernando Ortiz, de escritura sabrosa, picante, penetrante, bella y certera. 
Don Fernando establece la reflexión antropológica en Cuba, el ejercicio etnográfico, la preocupación por analizar la variedad cultural cubana, por crear conocimiento acerca de ella, por mostrar que es la columna vertebral de una sociedad que inicia su forja en el colonialismo. Con estas referencias, Victoria Novelo sitúa su texto en la tradición latinoamericana de hacer antropología, sin ignorar las contribuciones universales de la disciplina. Hemos de recordar que B. Malinowski, el antropólogo de la escuela británica de antropología social, comúnmente asociado con la empresa colonial inglesa, fue admirador de Fernando Ortiz, a quien reconoció aportaciones duraderas para la antropología; manifestó su interés por los textos del etnógrafo cubano y aprendió de él la tradición latinoamericana. Igual actitud tuvo Malinowski con Julio de la Fuente, el antropólogo mexicano con quien estudió los sistemas de mercado en Oaxaca y de quien recibió una influencia definitiva para reconocer la importancia de la historia en el análisis antropológico. El texto de Victoria Novelo es parte de esa tradición analítica que aborda nuestras realidades con mirada segura y con reflexión crítica.

El primer capítulo: "Yucatecos en Cuba”, discute la relación entre poblaciones y migraciones, y establece la importancia del tráfico de personas en la conformación del mosaico latinoamericano. Los yucatecos están en Cuba desde el siglo XVI, es decir, desde los albores del régimen colonial. Por supuesto, son los ma- yas yucatecos los que protagonizan aquel momento inicial. Uno aprende en este capítulo no sólo lo complejos que son la migración y los movimientos de población, sino cómo se fue forjando una frontera, un ámbito de convergencias, como hace años escribí de la frontera sur de México. Porque lo que nos enseña también este libro es que la Península de Yucatán es una frontera con el Caribe, formada desde tiempos anteriores a la Colonia y reafirmada cuando ésta se consolidó. En este capítulo, la autora empieza a elaborar una reflexión sobre Yucatán desde la perspectiva del proceso migratorio situado en los diferentes escenarios sociopolíticos y culturales, tanto de cubanos como de mexicanos. El texto se lee como una introducción para analizar la formación de las nacionalidades de América Latina y cómo se entrelazan.

Aspectos que me llaman la atención son la mención de los chichimecas y la impresionante debacle demográfica producida por el colonialismo. De aproximadamente 200000 habitantes que había en la isla de Cuba al llegar los españoles, la población bajó a 2000 a mediados del siglo XVI. Estamos hablando de exterminio. Acerca de los chichimecas, Novelo afirma que eran llamados "mecas" y "mecos", y llegan como prisioneros o como esclavos para ser empleados en la servidumbre doméstica. Es una pista que habrá que seguir. Además, en el primer capítulo la autora analiza el Caribe como un territorio donde el capitalismo inicia su expansión y su inter- nación en el continente americano. Se logra relacionar la migración yucateca a Cuba con esos procesos históricos ocurridos también en México. Hay un cúmulo de sugerencias en estos renglones para quienes estudian migraciones en la actualidad.

En el segundo capítulo, dedicado al siglo XX, Victoria Novelo escribe acerca de la Revolución Mexicana y uno de sus efectos en Yucatán: la migración de hacendados hacia $\mathrm{Cu}$ ba. Como sabemos, el sistema de haciendas yucateco fue peculiar, apoyado en relaciones de explotación de la mano de obra indígena que semejaron un sistema esclavista. El mote de casta divina quiere caracterizar a una de las más poderosas oligarquías de México. Miembros de este círculo de poder fueron a vivir a Cuba cuando la Revolución Mexicana introdujo la reforma agraria y otros cambios. Entre 1914 y 1917 se produjo la mayor migración de yucatecos a Cuba. En 1922 asumió la gubernatura del estado Felipe Carrillo Puerto, el famoso gobernador socialista de Yucatán, y la casta divina se vio severamente afectada.

Entre quienes migran a Cuba en la década de 1930 está la poeta Rosario Sansores, casada con un cubano. Rosario Sansores es la autora de aquellos versos que todos hemos cantado en alguna ocasión y que dicen: "cuando tú te hayas ido, me envolverán las sombras", que pasa en ocasiones como una canción ecuatoriana porque un músico de esa nacionalidad le compuso la música. 


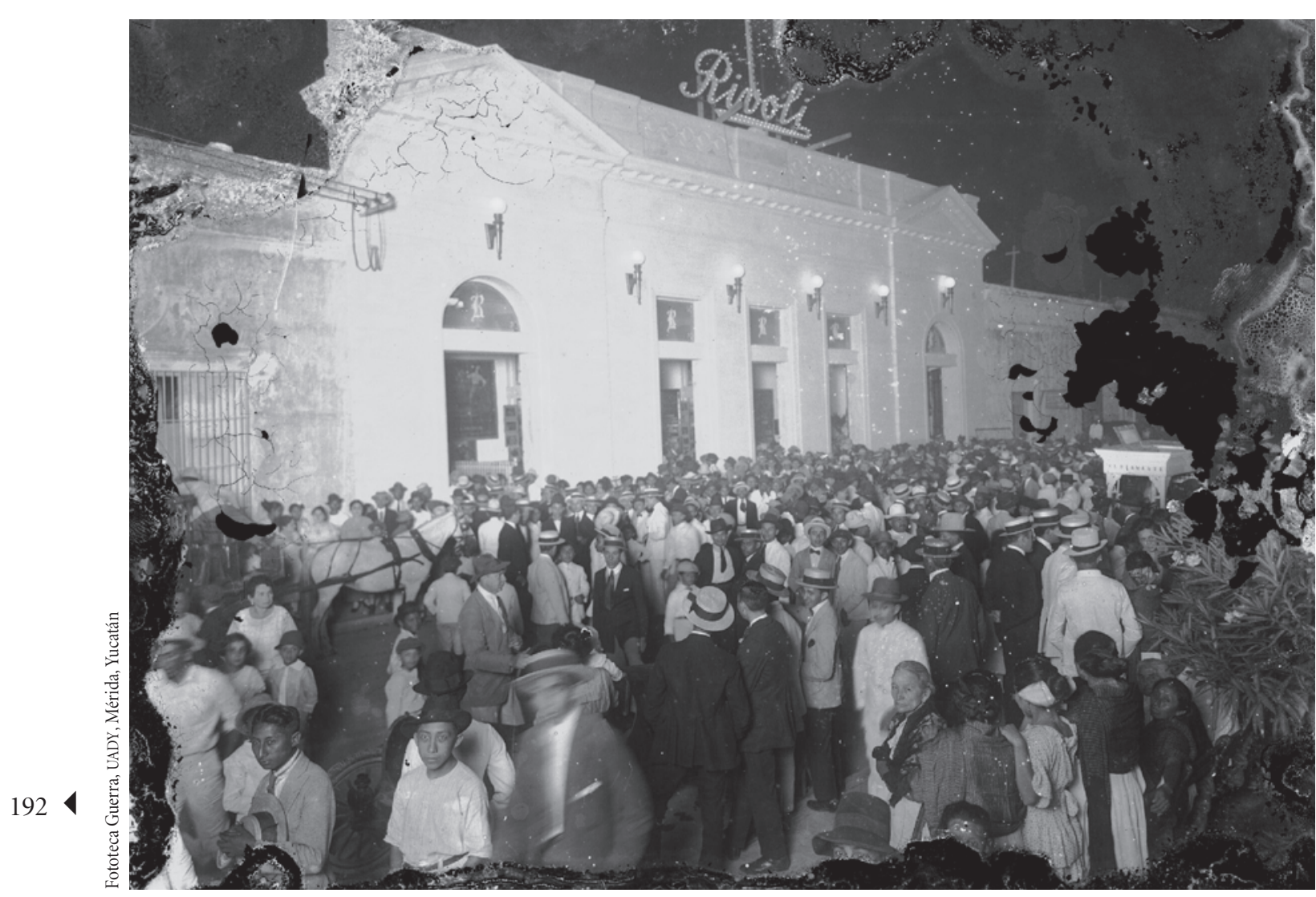

Multitud reunida a las puertas del cine Rívoli (hoy cine Rex), calle 57 por 70 y 72, barrio de Santiago, Mérida, ca. 1920.

Igualmente, la poeta tabasqueña Manuela Azcaino, yucateca por adopción, emigró a Cuba. A la postre, ella sería una de las primeras en hacer extensas observaciones acerca de la migración de yucatecos a la isla. En este capítulo, Victoria Novelo aborda el aspecto de los lugares de residencia de los yucatecos en la Cuba de aquella época, informándonos que el centro de la ciudad de La Habana fue el sitio que mayor concentración presentó.

El capítulo tercero de Yucatecos en Cuba aborda a los que se quedaron. Así, nos enteramos de la existencia de la Loma del Grillo como último reducto del territorio étnico mexicano en la Isla Bella. Pero también, de la existencia de los "yucatecos coreanos", a quienes Victoria Novelo dedica un apartado que incluye una interesante foto publicada en la página 119. El periplo coreano es descrito al detalle. Coreanos que dejaron su tierra apenas iniciado el siglo XX para trasladarse a Yucatán, y ya como yucatecos, terminar en Cuba. Recordé, por cierto, que actualmente existe una colonia de coreanos en Comitán. El capítulo cuatro se dedica a describir la situación de los que "iban y venían"; sobre todo músicos, deportistas, médicos y teatreros. Leyendo este capítulo, me vino a la memoria el caso de "los Moralitos", ese excelente grupo de músicos que vinieron a Chiapas en las épocas del Instituto Chiapaneco de Cultura y se quedaron en México. Como ése, hay varios casos en el relato de Victoria Novelo. Recordé que Benito Juárez estuvo de paso en Cuba, asunto sobre el que trabaja Mario Alberto Nájera. Aquí, en este capítulo, escrito con maestría, Novelo nos describe un entorno de frontera, esa frontera aún no percibida por el país, 
que es la caribeña. Frontera en la acepción sur: punto de convergencias, de múltiples intercambios, escenario de un "ir y venir" intenso. Recordé que ese "ir y venir" de mexicanos por el Caribe incluye la historia de "Las chiapanecas": navegando hacia Cuba la compañía de revistas de Guadalupe Rivas Cacho, sucedió que como parte de ella iba la marimba de los Hermanos Gómez. Juan Arozamena, esposo de Rivas Cacho, escuchaba "enronado" a la marimba, compuso en esa nave que se dirigía a La Habana la música y letra de "Las chiapanecas", que fue incluida en el repertorio de la compañía. De manera que uno de los himnos de los chiapanecos fue compuesto en aquella frontera caribeña, en ese mare nostrum que es el Caribe, y estrenado en 1924 en La Habana, en el teatro Variedades Payret. Por cierto, en tierra mexicana, "Las chiapanecas" fue estrenada por los propios Hermanos Gómez en el teatro "Progreso" de la ciudad de Mérida, Yucatán. Fue hasta 1930 que se estrenó en Tuxtla Gutiérrez, un 2 de diciembre, ejecutada por la misma marimba en el teatro Emilio Rabasa.

El mismo capítulo describe las mutuas influencias entre músicos cubanos y mexicanos, un tema que aún permite decir más. Lo mismo en el deporte, la medicina, el teatro. En la página 129 encontramos una foto de César Portillo de la Luz y Vicente Garrido, publicada por vez primera en el Granma el 10 de enero de 1991. Incluso, Victoria Novelo menciona el caso de las cabareteras cubanas, que causaron revuelo cuando llegaron removiendo las hormonas de los caballeros yucatecos. Escribe Novelo: "su presencia es recordada por la conmoción que causaron entre los varones yucatecos y, por ende, en sus familias.

Mencionaré también el caso del béisbol, que los cubanos introducen a Yucatán junto a las tertulias de café. Éste fue un aporte duradero. Los Venados de Yucatán han sido campeones de la Liga Mexicana en varias ocasiones y las reuniones alrededor de una buena taza de café siguen siendo parte de la vida meridiana. Bien menciona la autora los trabajos del académico cubano Félix Julio Alfonso López, quien nos ha visitado en Chiapas en varias ocasiones. Uno de los libros más recientes de Félix se titula Con las bases llenas. Béisbol, historia y revolución (Editorial Científico-Técnica, La Habana, 2008). Y menciona también como una lectura igualmente interesante el libro de James D. Cockroft, Latinos en el béisbol (Siglo XXI, México, 1999). Igualmente interesante resulta la reflexión sobre el estereotipo charro como modelo de lo "mexicano" que se difundió en Cuba y, de hecho, en el mundo. Lo cierto es que esa imagen fue parte del esfuerzo del Estado Nacional Mexicano por crear una sociedad monocultural. Al igual que en Cuba, en las salas de cine de la ciudad de Tuxtla Gutiérrez, allá por la década de 1950, se exhibía a los charros de los Altos de Jalisco como el prototipo de los mexicanos.

En el capítulo cinco, Victoria Novelo escribe acerca de la imagen de
Cuba en los migrantes. ¿Quién no recuerda a Tres Patines y Nana $\mathrm{Ni}$ na? Durante décadas, ese programa de radio - "La tremenda corte"transmitió una imagen de Cuba que no sólo compartieron los migrantes sino los mexicanos. Es un capítulo de interés para discutir asuntos tan complicados como el de la identidad, el lugar de la gastronomía en la forja de la misma, la memoria de la tierra nativa. Victoria Novelo borda un texto espléndido alrededor de una pregunta: ¿se parecen Yucatán y Cuba? Invito a explorar ese texto.

En las reflexiones finales, resalta el tema de la existencia de una frontera, de un mar, que une pero cambia para establecerse como barrera, al transformarse la sociedad cubana con la Revolución. Mucho hay que meditar sobre esta reflexión. La frontera está en la naturaleza de las sociedades y no en los ámbitos geográficos. En ese sentido, permanece el mutuo reconocimiento de mexicanos y cubanos de que, encima de todo, persiste una añeja tradición de compañía entre ambos pueblos.

El libro se completa con apéndices que traslucen el trabajo intenso de la autora para documentar su texto. El glosario resulta muy útil para percibir la dinámica de los intercambios idiomáticos entre ambos pueblos. La bibliografía es una fuente de información para quien desee continuar explorando en el Caribe una de las raíces del Estado Nacional Mexicano. 